\title{
DENSIDAD Y ANATOMIA DE LA MADERA EN FAMILIAS MEJORADAS DE SAUCES EN ARGENTINA ${ }^{1}$
}

\author{
Silvia Monteoliva ${ }^{2}$ e Teresa Cerrillo ${ }^{3}$
}

\begin{abstract}
RESUMEN - El objetivo del trabajo fue la evaluación de densidad y anatomía de la madera en siete familias de sauces, con vistas a seleccionar clones aptos para la producción de madera para usos sólidos y papel. Se cruzaron progenitores de cinco especies de sauces (S. babylonica, S. alba, S. nigra, S. amygdaloides y $S$. matsudana), obteniéndose 1800 individuos producto de cruzamientos controlados y polinización abierta, dentro del programa de mejoramiento de sauces del INTA. A los 34 meses se efectuó una primera selección por criterios de crecimiento, sanidad y forma. De esta primera fase de mejoramiento se seleccionaron 218 genotipos sobre los cuales se evaluaron las características anatómicas cuantitativas y la densidad de la madera. Los resultados indican que ninguna familia presenta buenos resultados en todas las propiedades. En una selección priorizando el vigor, la familia 08.09 presentó las siguientes características: buen crecimiento en diámetro $(6,1 \mathrm{~cm})$, fibras largas y de pared gruesa $(850 \mu \mathrm{m}$ y $2,22 \mu \mathrm{m})$, pocos vasos $\left(94 . \mathrm{mm}^{-2}\right)$ y densidad intermedia a baja $\left(350 \mathrm{~kg} \cdot \mathrm{m}^{-3}\right)$. Priorizando el rendimiento, material fibroso e indirectamente la resistencia, las familias 08.01 y 08.07 presentaron: densidad relativamente alta $\left(403-397 \mathrm{~kg} \cdot \mathrm{m}^{-3}\right)$, fibras largas $(836-864 \mu \mathrm{m})$, vasos pequeños $(46-45 \mu \mathrm{m})$ y bajo crecimiento en diámetro $(3-2,85 \mathrm{~cm})$. Los cruzamientos donde intervienen las especies Salix nigra, $S$. amygdaloides y $S$. matsudana se destacaron ya que presentan buenos crecimientos y las mejores combinaciones xilológicas para diferentes destinos industriales.
\end{abstract}

Palabras clave: Salix; Calidad de madera; Mejoramiento.

\section{DENSIDADE E ANATOMIA DA MADEIRA EM FAMÍLIAS MELHORADAS DE SALGUEIROS NA ARGENTINA}

\begin{abstract}
RESUMO - O objectivo deste estudo foi a avaliação da densidade e anatomia da madeira em sete famílias melhoradas de salgueiro, de modo a selecionar os clones apropriados para a produção de madeira sólida e papel. Progenitores de cinco espécies de salgueiros foram cruzados (S. babylonica, S. alba, S. nigra, S. amygdaloides e S. matsudana), obtendo-se 1.800 individuos produto das hibridações controladas e de polinização aberta, dentro do Programa de Melhoramento de Salgueiro da INTA. Aos 34 meses, foi efetuada a primeira seleção, considerando-se os critérios: crescimento, sanidade e forma. Nesta primeira fase de melhoramento, 218 genótipos foram selecionados, em que foram avaliadas as características anatomicas quantitativas e a densidade da madeira. Os resultados indicaram que nenhuma familia teve bons resultados em todas as propriedades. Na seleção por crescimento, a família 08.09 apresentou as seguintes características: bom crescimento em diâmetro $(6,1 \mathrm{~cm})$, fibras longas e de paredes grossas $(850 \mu \mathrm{m}$ e 2,22 $\mu \mathrm{m})$, poucos vasos $\left(94 \mathrm{~mm}^{-2}\right)$ e baixa densidade $\left(350 \mathrm{~kg} \cdot \mathrm{m}^{-3}\right)$. Priorizando a seleção para rendimento, a produção de fibra e indiretamente a resistência, as famílias 08.01 e 08.07 apresentaram: densidade relativamente elevada (403-397 kg. $\mathrm{m}^{-3}$ ),

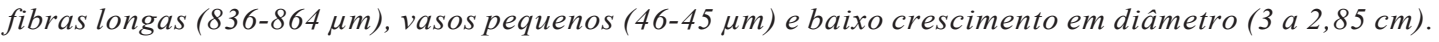
Os cruzamentos que envolveram a espécie Salix nigra, S. matsudana S $\boldsymbol{S}$. amygdaloides destacaram-se como de bom crescimento e as melhores combinações xilológicas para diferentes destinos industriais.
\end{abstract}

Palavras-chave: Salix; Calidade da madeira; Melhoramiento.

\footnotetext{
${ }^{1}$ Recebido em 12.06.2013 aceito para publicação em 23.10.2013.

${ }^{2}$ Facultad de Ciencias Agrarias y Forestales, Universidad Nacional de La Plata. CONICET. Argentina. E-mail: <smonteoliva@yahoo.com.ar>.

${ }^{3}$ Instituto Nacional de TecnologíaAgropecuaria (INTA), EEA Delta del Paraná, Campana, Argentina. E-mail: $<$ tcerrillo@gmail.com>.
} 


\section{INTRODUCCIÓN}

En Argentina la mayoría de las plantaciones de sauce (Salix spp.) están establecidas en el Delta del Paraná (provincias de Buenos Aires y Entre Ríos), donde se dan excelentes condiciones ecológicas y edáficas para la adaptabilidad del género. El sauce es de importancia central para la producción forestal de la región, constituyendo una fortaleza para el desarrollo territorial. Desde el punto de vista de las aplicaciones, el sauce posee aptitud para producción de madera, bioenergía, control de la erosión y fitorremediación. Actualmente, alrededor del $90 \%$ de la madera de sauce producido en el país se usa para fabricar papel y tableros de partículas. Sin embargo es también aplicable para "usos sólidos" (aserrado y debobinado en la primera transformación, y muebles), notándose en los últimos años un mayor interés en este sentido. De las 83.370 has de Salicáceas implantadas en toda la región, el $82.5 \%$ corresponden a sauces (BORODOWSKI, 2006, 2011), pero a la vez es significativa la falta de una masa crítica de calidad para aplicaciones sólidas. Esto enfatiza la necesidad de mejorar las plantaciones y, con tal fin, de contar con materiales selectos, sobre la base de volumen y calidad de la madera. En este sentido, el Instituto Nacional de Tecnología Agropecuaria, Argentina (INTA) desarrolla desde 2003 un programa de mejoramiento, principalmente enfocado al Delta, con el objetivo de obtener genotipos adaptados a la región. La estrategia considera la ampliación de la base genética, especialmente mediante cruzamientos inter e intraespecíficos, y la selección clonal (CERRILLO, 2005, 2009). La selección comienza a los 3 años de edad de los ortets con una selección en los bancos de progenies, del cual se ocupa el presente trabajo. Las etapas posteriores se efectuarán en los bancos clonales y en los ensayos a campo. Los criterios de selección considerados son: crecimiento, sanidad, forma y calidad de la madera (CERRILLO; ORTIZ, 2009). Se cuenta en la actualidad con más de 3000 genotipos en diferentes fases de selección. Parte de éstos, son los individuos de un banco de progenies de 1800 individuos originales (ortets) que dio lugar a la selección de 218 ramets, sobre los que trata el presente trabajo.

De todas las especies de Salix cultivadas en el Delta del Río Paraná, el sauce americano (Salix babylonica var. sacramenta) siempre se ha destacado por su largo de fibra, su relativa alta densidad y buena resistencia en las pulpas quimimecánicas (MONTEOLIVA,
2011). Pocas especies y/o clones introducidos han sido estudiados exhaustivamente con respecto a las propiedades de la madera deseables para lograr la difusión de otros clones con buena aptitud para diferentes industrias. Algunos trabajos de referencia incluyen algunas propiedades parciales anatómicas y de densidad (BONAVÍADE GUTHY RAGONESE, 1980; BONAVÍA DE GUTH, 1981, 1982, 1987; SENISTERRA et. al., 2000; MONTEOLIVA et. al., 2002). En estos trabajos se reportan descripciones xilológicas y valores promedios para diferentes clones en la edad de rotación (10 años), que pueden sintetizarse de la siguiente manera: las fibras son de tipo libriforme con longitudes que pueden variar entre $800-1200 \mu \mathrm{m}$, con espesores de pared entre 1,9-3,3 $\mu \mathrm{m}$, los vasos son solitarios y múltiples cortos, pequeños (55-80 $\mu \mathrm{m}$ de diámetro) y muy numerosos (80-100. $\left.\mathrm{mm}^{-2}\right)$, con placas de perforación simple, la densidad básica de la madera oscila entre 300-420 $\mathrm{Kg} \cdot \mathrm{m}^{-3}$. Evaluaciones más completas pueden hallarse en los primeros trabajos de nuestro grupo de investigación dirigidos hacia propiedades que influencian la calidad de la pulpas quimimecánicas con las que se produce actualmente el papel de diario (MONTEOLIVA et. al., 2005ab, 2007, 2008; MONTEOLIVA; MARLATS, 2007; MONTEOLIVA, 2009, 2011). Para algunos clones de sauces se han calculado heredabilidades de la densidad de la madera y longitud de fibras hallándose altos valores para estas dos características de la madera (MONTEOLIVA et. al., 2005a). Estos antecedentes indican que la elección de buenos progenitores en los cruzamientos generados en los programas de mejoramiento podría derivar en progenies con propiedades de la madera mejoradas para distintos destinos industriales (BONAVÍA DE GUTH, 1987; CERRILLO; ORTIZ, 2009). No existen evaluaciones de propiedades mecánicas en los nuevos clones de sauces, por este motivo se toma a la densidad como indicador de calidad y resistencia en los productos sólidos (PANSHIN; DE ZEEUW, 1980; BARNETT; JERONIMIDIS, 2003).

El objetivo del trabajo fue la evaluación de la densidad y anatomía de la madera en familias de sauces (Salix) producto de la etapa inicial de selección del programa de mejoramiento de sauces en Argentina, con vistas a seleccionar clones mejorados aptos para la producción de madera de calidad para usos sólidos (aserrado y debobinado) y papel para diarios. 


\section{MATERIALES Y MÉTODOS}

En el marco del programa de mejoramiento de sauce del INTA, en el año 2008 se efectuó una primera selección sobre un banco de progenies de sauce integrado por 1800 ortets o individuos producto de semilla. Estos individuos corresponden a siete familias en las que se cruzaron de las especies: Salix alba L., $S$. amygdaloides Anderss., S. babylonica L., S. matsudana Koidtz y S. nigra Marsh. Cinco de las siete familias fueron obtenidas mediante hibridaciones controladas en invernadero; las otras dos familias, 08.05 y 08.09, se originaron por polinización abierta de madres destacadas por sus características forestales (crecimiento, sanidad y forma). En la tabla 1 se indican las diferentes combinaciones con los orígenes y procedencias de cada progenitor.

La elección de los progenitores fue realizada sobre la base de las características de vigor y características de la madera (densidad y longitud de fibra). Las especies, S. nigra y S. amigdaloides presentaron los mejores crecimientos tanto en diámetro como en altura en evaluaciones tempranas (CERRILLO; ORTIZ, 2009), mientras que $S$. alba y $S$. matsudana tuvo buenos crecimientos (aunque menores que las anteriores) con buenas características de las fibras y densidad de la madera intermedia (360-390 Kg. $\left.\mathrm{m}^{-3}\right)$. S. babylonica presentó la mejor densidad y longitud de fibras en su madera (densidad superior a $400 \mathrm{Kg} \cdot \mathrm{m}^{-3}$ y fibras mayores a $1000 \mu \mathrm{m}$ ) y los peores crecimientos (BONAVÍA; RAGONESE, 1980; BONAVÍADE GUTH, 1982, 1987; SENISTERRAETAL., 2000; MONTEOLIVA ET AL., 2002).

A los 10 meses de edad, las 1800 progenies se plantaron a campo, aplicándose un diseño completamente aleatorizado, 10 individuos/parcela/ familia y, repeticiones variables según la cantidad total de individuos por familia, constituyendo un banco de progenies. A los 34 meses se registró sobrevivencia (1674 individuos vivos) y se midieron las alturas de todos los genotipos, registrándose datos de afecciones corticales y forma del ejemplar. La observación de sanidad consideró síntomas y/o signos de las principales enfermedades foliares (roya, Melampsora spp. y antracnosis, Marsonina salicicola) y de corteza (cancrosis, ocasionada por agentes fúngicos y/o bacterianos). Sobre la base de la variable crecimiento en altura, previo rechazo de todos los ortets que presentaban cancros en sus fustes, se efectuó un "screening" seleccionándose 218 genotipos (potenciales clones) que se establecieron en un banco clonal a campo, a razón de 5 estacas por clon.

La experiencia se localizó en la Estación Experimental Agropecuaria (EEA) Delta del Paraná del INTA, situada en la margen izquierda del Río Paraná en su intersección con el Canal Laurentino Comas, Partido de Campana, Provincia de Buenos Aires, al sur del Delta del Río

Tabla 1 - Material genético evaluado: origen de cada familia.

Tabela 1 - Material genético avaliado: origem de cada família.

\begin{tabular}{|c|c|c|c|}
\hline Familia & Cruza-miento & Progenitores & Origen del progenitor/Procedencia \\
\hline 08.01 & $\mathrm{CC}$ & $\begin{array}{l}\text { madre: } S \text {.matsudana SI64-012 } \\
\text { padre: } S . \text { babylonica SE81-001 }\end{array}$ & $\begin{array}{l}\text { Beijing, China / Istituto de Pioppicoltura. Italia } \\
\text { Nanjing, China/ Istituto de Pioppicoltura. Italia }\end{array}$ \\
\hline 08.02 & $\mathrm{CC}$ & $\begin{array}{l}\text { madre: } S . \text { alba } \text { S-59-005 } \\
\text { padre: } \text { S. babylonica } \text { SE } 81-001\end{array}$ & $\begin{array}{l}\text { Valenza Po, Italia/ Istituto de Pioppicoltura. Italia } \\
\text { Nanjing, China/ Istituto de Pioppicoltura. Italia }\end{array}$ \\
\hline 08.03 & $\mathrm{CC}$ & $\begin{array}{l}\text { madre: } S . \text { alba } \mathrm{S}-59-005 \\
\text { padre: (S. matsudana } x \text { S. alba) } 97.11 .01\end{array}$ & $\begin{array}{l}\text { Valenza Po, Italia/ Istituto de Pioppicoltura. Italia } \\
\text { CIEF, Argentina / INTA Argentina }\end{array}$ \\
\hline 08.05 & $\mathrm{CC}$ & $\begin{array}{l}\text { madre: } \text { S. amygdaloides CAN690 } \\
\text { padre: S. alba } \mathrm{S} 1\end{array}$ & $\begin{array}{l}\text { Texas, USA / Toronto University, Canada } \\
\text { Istituto Novi Sad, exYugoslavia/ Servicio de } \\
\text { Investigación Agraria, España }\end{array}$ \\
\hline 08.06 & $\mathrm{CC}$ & madre: S. amygdaloides CAN 690 & Texas, USA/ Toronto University, Canada \\
\hline 08.07 & $\mathrm{PA}$ & $\begin{array}{l}\text { padre: S. babylonica SE81-001 } \\
\text { madre: S. matsudana NZ 695padre: ? }\end{array}$ & $\begin{array}{l}\text { Nanjing, China/ Istituto de Pioppicoltura. Italia } \\
\text { China / Forest Research Institute, New Zealand }\end{array}$ \\
\hline 08.09 & PA & $\begin{array}{l}\text { madre: (S. matsudana } x \text { S. nigra) } \\
\text { 98.13.08 } \\
\text { padre: ? }\end{array}$ & CIEF, Argentina / INTA Argentina \\
\hline
\end{tabular}

CC: cruzamiento controlado; y PA: polinización abierta. 
Paraná. El Delta es un amplio mosaico de humedales ubicado entre los $32^{\circ} 5^{\prime}$ y $34^{\circ} 29^{\prime}$ de latitud sur y $60^{\circ}$ $45^{\prime}$ de longitud oeste, al sur de la Mesopotamia argentina. El Río Paraná, en su recorrido de 4.000 km drena una cuenca de una superficie de $2.600 .000 \mathrm{~km}$; en su curso inferior se registra una ampliación del valle fluvial, conformando el delta, de aproximadamente 1.750.00 (Figura 1).

En terrenos del INTA Delta, el Banco de Progenies se instaló en un terreno bajo, protegido por endicamiento y drenado mediante un sistema de zanjas y canales, que no permite el ingreso de crecientes del río. Los suelos son de origen aluvional.

Datos meteorológicos para la zona de ensayo (serie 1976-2005): temperatura media $16,4^{\circ} \mathrm{C}$; temperatura mínima absoluta $-5^{\circ} \mathrm{C}$; temperatura máxima absoluta $39^{\circ} \mathrm{C}$; precipitación anual media 1.127,2 mm; precipitación anual máxima media 1.530,3 mm y precipitación mínima media 796,8 mm. El área donde se realizó la experiencia registró una alta concentración de lluvias ocurridos en la fase de implantación del Banco de Progenies entre octubre de 2009 y mayo de 2010 (1252mm). Este fenómeno, combinado con los elevados niveles del río Paraná, dificultaron la evacuación del agua del campo por gravedad a través de la compuerta, originando una gran acumulación en el terreno en un lapso de aproximadamente 20 días (el 15 de enero de 2010),

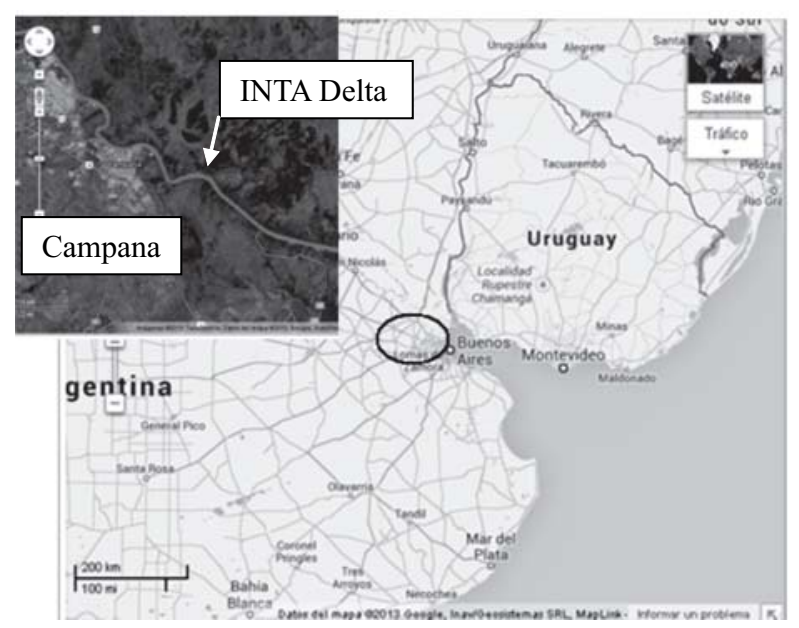

Figura 1 - Mapa de ubicación de la zona de ensayo (INTA Delta, Argentina).

Figura 1 - Mapa de localização da área de ensaio (INTA Delta, Argentina). alcanzando el agua una altura máxima aproximada de $80 \mathrm{~cm}$; lo cual ocasionó pérdidas en la plantación inicial del banco de Progenies.

\section{Evaluación de la madera}

La madera de los 218 clones experimentales selectos fue analizada con el objetivo de sumar elementos para continuar la mejora sobre la base del criterio de calidad de la madera.

Se tomó una muestra destructiva (rodaja) en la base de cada ejemplar, de 5-7 cm de espesor. Se registraron los diámetros con y sin corteza (medición de 2 diámetros opuestos).

Se determinaron las siguientes variables: densidad básica, morfometría de fibras (longitud y espesor de pared) y de vasos (diámetro y frecuencia).

Las rodajas fueron descortezadas para la determinación de densidad básica. La densidad se determinó por el cociente entre peso seco y volumen saturado.

Longitud de fibras: se tomaron dos astillas del último anillo de crecimiento de cada rodaja (año 3) para hacer macerados y medir longitud de fibras. Se realizaron disociados con ácido acético y agua oxigenada en partes iguales (FRANKLIN, 1945). Se realizaron preparados histológicos transitorios sin teñir con la suspensión de fibras.

Espesor de pared de fibras, diámetro y frecuencia de vasos: se realizaron cortes histológicos transversales teñidos con safranina ( $1 \%$ ) sobre probetas cortadas del último anillo de crecimiento (año 3). La observación de los mismos se realizó con microscopio óptico Olympus (4x, 20x y 40x).

Se tomaron imágenes digitales de los preparados. Las fibras y vasos se midieron sobre dichas imágenes con el programa Image Tool (versión libre). Se midieron 100 fibras y vasos por clon, para frecuencia de vasos se contaron la cantidad de vasos sobre 5 imágenes (cada imagen con un área de $4 \mathrm{~mm}^{2}$ ) y se calculó el número por $\mathrm{mm}^{2}$.

Se realizó unANOVA simple con Familia como fuente de variación. El contraste de medias se realizó con la prueba de Tukey $(\mathrm{p}<0,05)$. Se utilizó el programa estadístico InfoStat (2005). Se realizó una selección de familias con mejor calidad de la madera.

Revista Árvore, Viçosa-MG, v.37, n.6, p.1183-1191, 2013 


\section{RESULTADOS}

Los análisis de la varianza indicaron que la familia resultó una fuente significativa de variación para todas las variables de la madera analizadas $(p<0,05)$.

La tabla 2 presenta los resultados promedio por familia para las variables longitud de fibras, espesor pared de fibras, diámetro y frecuencia de vasos y densidad básica. La prueba de Tukey indicó que hay diferencias significativas entre las medias de algunas familias.

Los crecimientos en diámetro y altura indicaron que las familias 05, 06 y 09 presentaron los mayores valores comprendidos entre 5,13-6,03 cm de DAP y 6,35-7,20 m de altura (datos no mostrados, CERRILLO; MONTEOLIVA, 2012).

Sin embargo, este crecimiento no fue acompañado por las mejores características de la madera. Las familias 05, 02 y 06 presentaron los menores valores de longitud de fibras, dos de las cuales tienen como progenitor femenino a $S$. amygdaloides. Por otro lado, la familia 03 presentó longitudes de fibras intermedias. Finalmente las familias 01, 07 y 09 presentaron las mayores longitudes de fibras, teniendo las tres a $S$. matsudana como progenitor femenino (Tabla 2, Figura 2).

Las familias 05, 06 y 09 presentaron los menores valores de densidad. Las mayores densidad las presentan las familias 01 y 07, ambas conformadas por S. matsudana, la primera de ellas por cruzamientos controlados con S. babylonica y la segunda familia fue obtenida por polinización abierta (Tabla 2, Figura 2).

Ninguna Familia presenta buenos resultados en todas las variables anatómicas y densidad. El crecimiento en diámetro presentó correlación negativa con la densidad de la madera $(r=-0,52)$ y positiva con el diámetro de vasos $(r=0,69)$. Por lo tanto, sobre la base del material genético estudiado, no es posible seleccionar clones con buen diámetro y alta densidad.

\section{Selección de Familias en la segunda etapa (banco clonal):}

En una selección priorizando el vigor, la Familia 08.09 (S. matsudana x S. nigra "98.13.08" x ?) presenta las siguientes características: Tabla 3.

En una selección priorizando el rendimiento, material fibroso y resistencias las Familias $\mathbf{0 8 . 0 1}$ (S. matsudana $x$ S. babylonica) y 08.07 ( $S$. matsudana $\mathrm{x}$ ?) presentan las siguientes características: Tabla 4 .

\section{DISCUSIÓN}

Los crecimientos en diámetro y altura indicaron que las familias 05, 06 y 09 presentaron los mayores valores comprendidos entre 5,13-6,03 cm de DAP y 6,35-7,20 m de altura (CERRILLO; MONTEOLIVA, 2012). Sin embargo, este crecimiento no fue acompañado por las mejores características de la madera como son: densidad relativamente alta (valores superiores a 400 $\mathrm{Kg} . \mathrm{m}^{-3}$ ), fibras largas y de buen espesor de pared (valores superiores a $800 \mu \mathrm{m}$ de longitud y 2,20 $\mu \mathrm{m}$ de espesor pared), vasos pequeños en diámetro y numerosos (hasta $45 \mu \mathrm{m}$ diámetro y más de 100 vasos. $\mathrm{mm}^{-2}$ ). Estos valores de referencia son indicados como valores umbrales mínimos para obtener una buena calidad de pulpa quimimecánica a partir de clones de sauces (REPETTI et. al., 1981; MONTEOLIVA et. al., 2005b). Para los destinos de madera sólida (aserrado o madera redonda) no hay referencias nacionales que permitan analizar las mejores combinaciones de propiedades en la materia prima a mejorar. Sin embargo la densidad de la madera es un muy buen indicador de calidad frente a estas

Tabla 2 - Medias ( \pm DS) por familia para todas las variables medidas en la madera a los 3 años de edad. Tabela 2 - Média $( \pm D P)$ por família das características avaliadas da madeira aos 3 anos de idade.

\begin{tabular}{|c|c|c|c|c|c|c|}
\hline Familia & $\mathrm{N}^{\mathrm{o}}$ clones & Longitudfibras & Espesor paredfibras & Diámetro vasos & Frecuencia vasos & Densidadbásica \\
\hline & & $\mu \mathrm{m}$ & $\mu \mathrm{m}$ & $\mu \mathrm{m}$ & $\mathrm{N}^{\mathrm{o}} \cdot \mathrm{mm}^{-2}$ & $\mathrm{~kg} \cdot \mathrm{m}^{-3}$ \\
\hline 08.01 & 56 & 836 d $( \pm 107)$ & 2,03 a $( \pm 0,67)$ & 46,2 a $( \pm 6,82)$ & $107 \mathrm{ab}( \pm 17)$ & $403 \mathrm{~b}( \pm 22)$ \\
\hline 08.02 & 4 & 788 a $( \pm 122)$ & $2,27 \mathrm{c}( \pm 0,42)$ & 44,2 a $( \pm 7,21)$ & $107 \mathrm{ab}( \pm 22)$ & $381 \mathrm{ab}( \pm 38)$ \\
\hline 08.03 & 5 & 816 b $( \pm 111)$ & $2,28 \mathrm{c}( \pm 0,38)$ & 43,1 a $( \pm 7,22)$ & $116 \mathrm{~b}( \pm 19)$ & $374 \mathrm{ab}( \pm 11)$ \\
\hline 08.05 & 75 & $800 \mathrm{ab}( \pm 116)$ & 2,00 a $( \pm 0,37)$ & $60,3 \mathrm{c}( \pm 10,37)$ & 102 a $( \pm 13)$ & 347 a $( \pm 17)$ \\
\hline 08.06 & 3 & $799 \mathrm{ab}( \pm 111)$ & 2,17 bc $( \pm 0,36)$ & 56,4 b $( \pm 9,19)$ & $110 \mathrm{ab}( \pm 10)$ & 327 a $( \pm 19)$ \\
\hline 08.07 & 10 & 864 e $( \pm 126)$ & 2,13 b $( \pm 0,37)$ & 45,5 a $( \pm 8,15)$ & $108 \mathrm{ab}( \pm 16)$ & 397 b $( \pm 22)$ \\
\hline 08.09 & 65 & 850 de $( \pm 122)$ & $2,22 \mathrm{c}( \pm 0,42)$ & $59,8 \mathrm{c}( \pm 11,18)$ & 94 a $( \pm 21)$ & 350 a $( \pm 17)$ \\
\hline
\end{tabular}

Letras distintas indican diferencias significativas $(\mathrm{p}<0,05)$.

$\mathrm{N}$ del banco clonal: 218 individuos. 


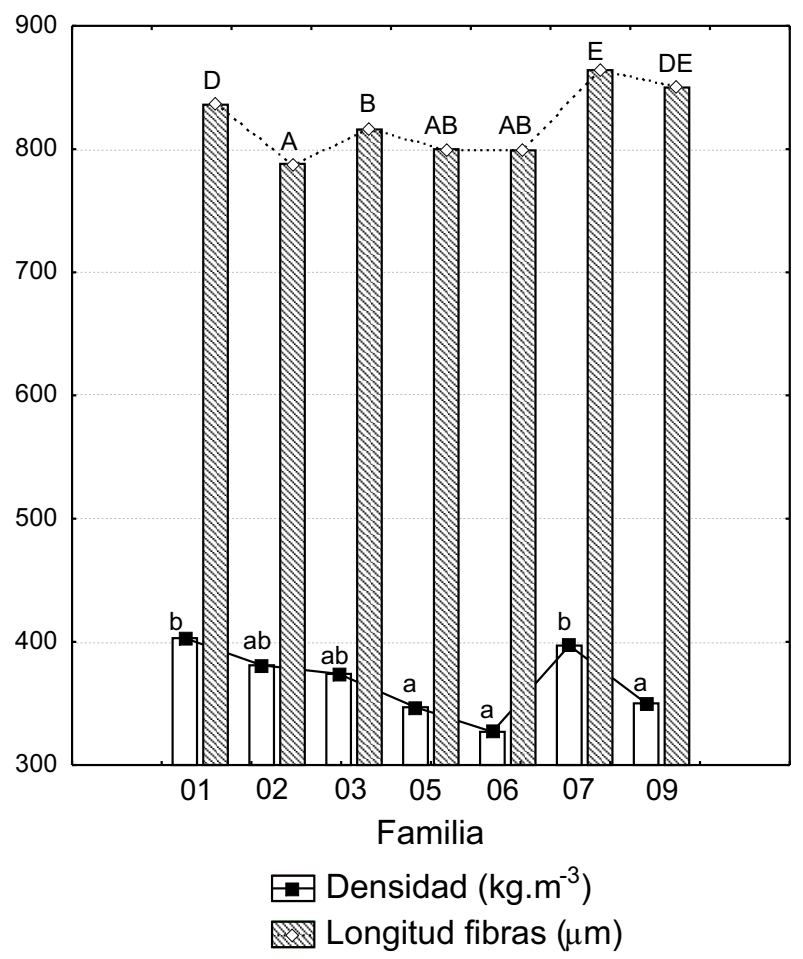

Figura 2 - Densidad básica y longitud de fibras promedios por Familia. Letras mayúsculas indican prueba de Tukey para longitud de fibras y minúsculas para densidad.

Figura 2 - Densidade básica e comprimento médio das fibras por família. Letras maiúsculas indicam teste de Tukey para o comprimento das fibras e letras minúsculas, para a densidade. aplicaciones ya que está positivamente correlacionado con las resistencias (BARNETT; JERONIMIDIS, 2003; PANSHIN; DE ZEEUW, 1980). Los diferentes clones de sauces actualmente en cultivo en el Delta brindan madera con densidades entre 350-400 Kg.m ${ }^{-3}$ (BONAVÍA; RAGONESE, 1980; SENISTERRA et. al.,2000). Obtener nuevos clones con densidades cercanas a $400 \mathrm{Kg} . \mathrm{m}^{-3}$ a edades tempranas, asegura que esta propiedad resulte elevada en la edad de turno, que para el caso de madera con destino a aserrado será mayor que la destinada a pulpa.

Ninguna Familia presenta buenos resultados en todas las variables anatómicas y densidad. Por las correlaciones obtenidas entre variables, y sobre la base del material genético estudiado, no es posible seleccionar clones con buen diámetro y alta densidad simultáneamente. La densidad de la madera y el espesor de pared de las fibras están relacionados positivamente con el rendimiento en pulpa y con las propiedades mecánicas en la madera sólida. La longitud de fibras es una característica altamente heredable y está relacionada con las resistencias mecánicas de los papeles. Asimismo, un diámetro de vasos mayor, permite una buena penetración de los licores de impregnación pero disminuye el rendimiento en pulpa (REPETTI, 1983; DEKA et. al., 1994; ARREGHINI; CERRILLO, 1996).

Según los resultados del trabajo se proponen dos tipos diferentes de selección: por vigor o por calidad de materia prima. Ambas selecciones tienen como progenitor femenino a Salix matsudana, demostrándose

Tabla 3 - Características distintivas de la Familia 08.09.

Tabela 3 - Características distintivas da Família 08.09.

\begin{tabular}{cc}
\hline Buena calidad & Mala calidad \\
\hline Alto crecimiento en diámetro & Baja densidad de la madera \\
Alta longitud de fibras & \\
Bajo número de vasos $/ \mathrm{mm}^{2}$ & \\
Alto espesor de pared de fibras & \\
\hline
\end{tabular}

Tabla 4 - Características distintivas de la Familias 08.01 y 08.07.

Tabela 4 - Características distintivas das Famílias 08.01 e 08.07.

\begin{tabular}{cc}
\hline Buena calidad & Mala calidad \\
\hline Alta densidad & Bajo crecimiento en diámetro \\
Alta longitud de fibras & \\
Bajo diámetro de vasos & \\
\hline
\end{tabular}

Revista Árvore, Viçosa-MG, v.37, n.6, p.1183-1191, 2013 
nuevamente que este genotipo presenta las mejores combinaciones de propiedades para diferentes destinos industriales (BONAVIADE GUTH, 1987; VASCHETTO, 1995; VASCHETTO; GRANDE, 2005). BONAVÍADE GUTH (1987) demostró que los descendientes de cruzamientos en los que intervenían como progenitores S. matsudana y S. nigra heredaban las buenas características de la madera, medidas en términos de densidad y longitud de fibras, así como también los buenos crecimientos en diámetro. En cambio, los cruzamientos con S. babylonica no heredan las buenas propiedades del leño de este genotipo. Vaschetto y Grande (2005) en la evaluación de calidad de pulpas sobre 16 clones mejorados demostraron la supremacía del genotipo $S$. matsudana frente a otras combinaciones. Se seleccionaron en esa oportunidad 9 clones, 5 de los cuales fueron cruzamientos $\mathrm{x}$ matsudana, que presentaron aptitud papelera similar o superior al sauce americano usado como testigo, en términos de índice de rasgado, tracción y coeficiente de dispersión.

Para la especie S. amigdaloides, que interviene en otro cruzamiento de las Familias seleccionadas, no se encontraron antecedentes de evaluaciones de la madera ni de las pulpas.

Las correlaciones juvenil-adulto y heredabilidades determinadas en otros trabajos para sauces (TAYLOR, 1975; DEKA et. al.,1994; ARREGHINI; CERRILLO, 1996; MONTEOLIVA et. al.,2005a) indican que algunas propiedades como longitud de fibras, espesor de pared y densidad presentan alta heredabilidad y sus valores aumentan con la edad. Mientras que los valores del tamaño y cantidad de vasos se estabilizan a edades muy tempranas (4-5 años). Por lo tanto es de esperar que a las edades próximas al turno (8-10 años para papel y superiores para madera sólida) los valores de estas variables aumenten.

\section{CONCLUSIONES}

En concordancia con resultados de evaluaciones precedentes de crecimiento en sauces, desde edad temprana se detectó la supremacía de los cruzamientos donde intervienen las especies Salix nigra, $S$. amygdaloides y S. matsudana.
Esta metodología de evaluación temprana de caracteres de la madera sería adecuada para utilizarla en una segunda etapa de selección donde se incorporen las propiedades de la madera que tienen efecto en la calidad de madera con diferentes destinos industriales. Es posible realizarlas con metodología no destructiva (muestras de barreno).

\section{AGRADECIMIENTOS}

A la Técnica Agr. Stella Ortiz por la colaboración en la obtención de los cruzamientos controlados y la selección; al personal de campo de la EEA Delta del Paraná del INTA, por el trabajo a campo en la instalación y selección de los materiales y a Papel Prensa SA por el aporte de datos meteorológicos del área y por su apoyo para las evaluaciones de madera.

\section{REFERENCIAS}

ARREGHINI, R.; CERRILLO, T. Los sauces en la Repúblcia Argentina. In: SESSION OF THE INTERNATIONAL POPLAR COMMISSION, 20., 1996, Budapest. Proccedings... Budapest: 1996. p.742-768.

BARNETT, J. R.; JERONIMIDIS, G. Wood quality and its biological basis. London: CRC Press, 2003.

BORODOWSKI, E. Álamos y sauces en el Delta del Paraná: situación del sector y silvicultura. In: JORNADAS ARGENTINAS DE SALICÁCEAS, 1 ., 2006, Buenos Aires. Jornadas... Buenos Aires: 2006. p.61-70.

BORODOWSKI, E. Estado de situación del cultivo de sauce en el Delta del Paraná. In: JORNADA TÉCNICA SOBRE EL SAUCE EN ELDELTA ENTRERRIANO, 2011. Acta... Buenos Aires: 2011.

BONAVÍA DE GUTH, E.; RAGONESE, A. Evaluación de las características del leño en relación a la calidad del papel de algunos híbridos de sauces obtenidos en Castelar (INTA). Buenos Aires: IDIA, 1980. p.25-30.

BONAVÍA DE GUTH, E. Características del leño de varias especies e híbridos de Salix en apoyo a la obtención de buenos clones papeleros. In: CONGRESO DE ATIPCA, 17., 1981, BuenosAires. Acta... BuenosAires: 1981. p.F25-F32.

Revista Árvore, Viçosa-MG, v.37, n.6, p.1183-1191, 2013 
BONAVÍA DE GUTH, E. Evaluación de varios híbridos obtenidos por cruzamiento de Salix alba $x$ S. matsudana. In: CONGRESO DE ATIPCA, 18., 1982, Buenos Aires. Acta... Buenos Aires: 1982. p.19-30.

BONAVÍA DE GUTH, E. Obtención y evaluación de nuevos clones de sauces y álamos en relación a su uso celulósico. In: CONGRESO DEATIPCA, 23., 1987, Buenos Aires. Acta...Buenos Aires: 1987. p.43-60.

CERRILlO, T. Panorama de los sauces en Argentina: avances en el mejoramiento genético y en la identificación. In: JORNADAS

ARGENTINAS DE SALICÁCEAS, 2., 2009, Mendoza. Disertación... Mendoza: 2009.

CERRILLO, T.; ORTIZ, S. Mejoramiento genético del sauce - Resultados de ensayos comparativos a edad temprana. In: CONGRESO FORESTAL MUNDIAL, 13., Buenos Aires, 2009.

Acta...Buenos Aires: 2009.

CERRILlo, T. Subprograma Sauces en el Delta. Mejores Árboles para Más Forestadores. PPMPM, Convenio SAGPyA-INTA. Proyecto Forestal de Desarrollo, 2005.

CERRILLO, T.; MONTEOLIVA, S. Evaluation of wood characteristics in the first selection phase of a willow (Salix spp) breeding program in Argentina. In: IUFRO CONFERENCE, 2012, Lisboa. Proceedings... Lisboa: 2012.

DEKA, G. C.; WONG, B. M.; ROY, D. N. Variation of specific gravity, fibre length and cell wall thickness in young Salix clones. Journal of Wood Chemistry and Technology, v.14., p.147-158, 1994.

FRANKLIN, G. L. Preparation of thin sections of synthetic resins and woodresin composites, and a new macerating method for wood. Nature, v.155, n.3924, p.51, 1945.

INFOSTAT/Prof..Sofware Estadístico. 1.1. Universidad de Córdoba. Estadística y Diseño. FCA, 2005.

MONTEOLIVA, S. et al. Estudio de la variación de la densidad básica en siete clones de Salix. Revista Facultad Agronomía La Plata, v.105, n.1, p.29-34, 2002.
MONTEOLIVA, S.; SENISTERRA, G.; MARLATS, R. Variation of wood density and fibre length in six willow clones (Salix spp.). IAWA Journal, v.26, p.197-202, 2005 a.

MONTEOLIVA, S. et al. Selección de clones de sauces por propiedades tecnológicas para la industria papelera. In: CONGRESO FORESTAL ARGENTINO (AFOA), 2005. Actas...

Corrientes: 2005b.

MONTEOLIVA, S.; MARLATS, R. Efecto del sitio, clon y edad sobre el crecimiento y la calidad de madera en sauces de corta rotación.

Revista Investigación Agraria, Sistema y Recursos Forestales, v.16, n.1, p.15-24, 2007.

MONTEOLIVA, S.; AREA, M. C.; FELISSIA, F. CMP pulps of willows for newsprint. Part 1: Pulps evaluation. Cellulose Chemistry Technology, v.41, n.4/6, p.263-272, 2007.

MONTEOLIVA, S.; AREA, M.C.; FELISSIA,

F. CMP pulps of willows for newsprint. Part 2: relationships between wood characteristics and pulp properties.

Cellulose Chemistry Technology, v.42, n.1, p.45-59, 2008.

MONTEOLIVA, S. Propiedades de la madera y de las pulpas CMP de Salicáceas: Estado del arte en Argentina. In: JORNADAS

ARGENTINAS DE SALICÁCEAS, 2., 2009, Mendoza. Acta...Mendoza: 2009.

MONTEOLIVA, S. Madera de sauces para la producción de papel periódico. Berlín: Editorial Académica Española, 2011.

PANSHIN, A.; DE ZEEUW, C. Textbook of wood technology. London: McGraw-Hill Book Company, 1980.

REPETTI, R. et al. Pastas de alto rendimiento de sauces de corta duración. In: CONGRESO TÉCNICO SOBRE CELULOSA Y PAPELATIPCA, 17., 1981, Buenos Aires. Trabajos Técnicos... Buenos Aires: 1981.p.51-85. 
REPETTI, R. Pastas de alto rendimiento de sauces, incidencia de la morfología de las fibras en las propiedades del papel. $19^{\circ} \mathrm{In}$ : CONGRESO TÉCNICO SOBRE CELULOSA Y PAPEL, ATIPCA, 19., 1983, Buenos Aires. Trabajos Técnicos... Buenos Aires: 1983. p.25-31.

SENISTERRA, G. et al. Propiedades del leño en clones del género Salix utilizados en programas de mejoramiento genético con aplicación a la industria papelera. Yvyraretá, v. 10, p.93-95, 2000.
TAYLOR, F. Wood property difference between two stands of sycamore and black willow. Wood and Fiber, v.7, n.1, p.187-191, 1975.

VASCHETTO, J. Evaluación de pulpas de sauces. Buenos Aires: Informe interno de Papel Prensa, 1995.

VASCHETTO, J.; GRANDE, J. Evaluación de pulpas de sauces experimentales obtenidos por mejoramiento genético. Buenos Aires: Informe interno, Laboratorio Centralde Papel Prensa, 2005. 
